\title{
$N$-LEVEL ATOMS IN MULTIPLE LASER FIELDS, THE LONG AND THE SHORT OF IT
}

\author{
J.H. EBERLY \\ Department of Physics and Astronomy, University of Rochester \\ Rochester, NY 14627, USA
}

\section{Dedicated to Prof. Iwo Biatynicki-Birula on the occasion of his 60th birthday}

The interaction of multi-level atoms with several to many lasers simultaneously is examined in two regimes, the "short" regime of resonant atomic response to prescribed laser fields, and the "long" regime of resonant pulse propagation in which several to many laser pulses pass through an active medium composed of many-level atoms. Results obtained with I. Białynicki-Birula et al. in the short regime are reviewed, and new results in the long regime are described.

PACS numbers: $42.50 . \mathrm{Rh}, 42.50 . \mathrm{Hz}$

In the fifty-year period 1875-1925 optical studies of the interaction of radiation with matter, from infrared to uv frequencies, provided the fuel for the quantum revolution. Data from these studies first called attention to regularities in atomic and molecular spectra, anomalous aspects of the photoelectric effect and of the blackbody spectrum, the apparent existence of "negative" oscillators in absorption, etc. This was the first golden age of quantum optics, although the term quantum optics was naturally not in use at the time. After an interval of another fifty years, and beginning very approximately in 1975, the field of quantum optics has again been one of the most fruitful frontiers in physics. This second golden age arrived with the development of the tunable laser, which provided optical spectroscopists with the ability to manipulate atomic and molecular matter to a degree barely dreamed of in the preceding decades. These manipulations themselves became interesting to study and atoms became "laboratories" for fascinating micro-experiments.

In this note we focus attention on "short" and "long" aspects of the light-atom interaction arising in studies of some of these manipulations. An important element will be the "area" of a plane-wave optical laser pulse, which is now understood to be a more fundamental measure of its strength than are more conventional measures such as pulse energy, fluence and intensity. Pulse area is defined by the time 
integral from the distant past up to the present time of the space-time-dependent amplitude of the electric field of a pulse

$$
A(z, t) \equiv \int_{\text {past }}^{t} \Omega\left(z, t^{\prime}\right) \mathrm{d} \iota^{\prime},
$$

where $\Omega(z, t)$ is the so-called complex Rabi frequency associated with the light-matter interaction

$$
\hbar \Omega(z, t) \equiv 2 d \cdot e \mathcal{E}(z, t) .
$$

It is implied that the electric field of the light wave, polarized in the (possibly complex) direction $e$, is written $E(z, t)=e \mathcal{E}(z, t) \exp [-\mathrm{i}(\omega t-k z)]+$ c.c., and $d$ is the dipole transition matrix element of the atomic or molecular transition with which the light is resonant. It is clear from (2) that $\Omega$ can be thought of as the dipole interaction energy between the atom and the light field in frequency units. For simplicity we will take $\Omega$ to be real.

Pulse area is significant in the first place because it is numerically equal to the angle of rotation of the Bloch vector [1] of a two-level atom in a laser field (or the angle of rotation of an electron spin in a microwave field, etc.). Thus it measures the degree of excitation of an atom coherently, i.e., without losing track of any atomic dipole phase which may be induced by the exciting radiation. In this way it is superior to the more familiar measures of excitation such as upper level probability and inversion.

Now we will formulate the excitation question somewhat generally, considering a quantum system with $N$ energy levels and using as many laser fields as necessary. There is a Rabi frequency (2) for each dipole-allowed transition in an atom, and we are interested in the excitation of an upper atomic level through successive transitions via lower levels, possibly requiring the application of a number of lasers together, each with its frequency tuned to a distinct atomic resonance. This is sketched in Fig. 1, where two-level, three-level and $(N+1)$-level excitation sequences are indicated. Multi-laser and multi-level excitation provides an inter-
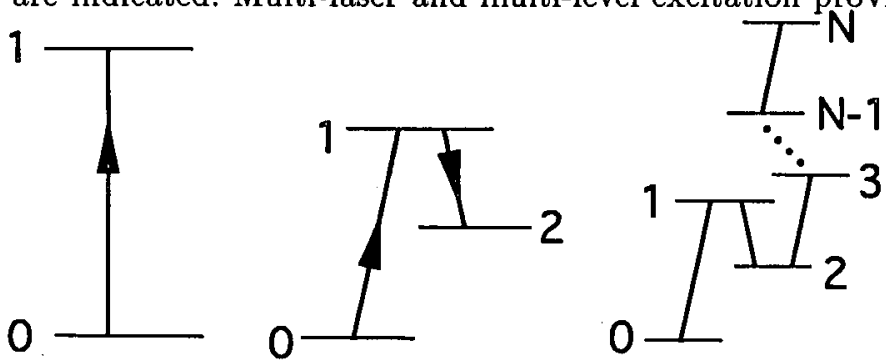

Fig. 1. Three $N$-level systems, showing exactly resonant laser excitation along a chain of dipole-allowed transitions.

esting mathematical problem because it allows mathematical methods developed in classical mechanics (for problems identified long ago by Euler, D'Alembert and Bernoulli) to be applied usefully to a quantum mechanical situation.

We assume that all transitions are exactly on resonance, and that Schrödinger's equation is written in the rotating wave approximation (RWA) [1]. We denote 
by $a_{m}$ the probability amplitude associated with the $m$-th level of the atom. The lasers are all assumed to be operating at constant field amplitudes. Then the equations for the $a$ 's can be written

$$
\mathrm{i} \frac{\mathrm{d}}{\mathrm{dt}}\left(\begin{array}{c}
a_{0} \\
a_{1} \\
\cdot \\
\cdot \\
\cdot \\
\cdot \\
a_{N}
\end{array}\right)=\frac{-1}{2}\left(\begin{array}{cccccc}
0 & \Omega_{1} & & & & \\
\Omega_{1} & 0 & \Omega_{2} & & & \\
& \Omega_{2} & 0 & \Omega_{3} & & \\
& & \Omega_{3} & & \cdot & \\
& & & \cdot & & \cdot \\
& & & & \cdot & \\
& & & & & .
\end{array}\right)\left(\begin{array}{c}
a_{0} \\
a_{1} \\
\cdot \\
\cdot \\
\cdot \\
\cdot \\
a_{N}
\end{array}\right) .
$$

Clearly, the determinant $D_{N}$ of the matrix is related to the oscillation frequencies for the amplitudes. This determinant is a Jacobi form and its minors satisfy the recurrence relation

$$
D_{m+1}+\lambda D_{m}+\left|\Omega_{m} / 2 \Omega_{1}\right|^{2} D_{m-1}=0 .
$$

In the late 1970's, with Z. Bialynicka-Birula, I. Białynicki-Birula and B.W. Shore, we studied these laser excitation problems both analytically and numerically for a number of special cases [2].

In a general situation, such as is represented by the Jacobi matrix appearing in (3), it is always helpful to heed advice attributed to Einstein, to make everything as simple as possible, but not more so. Two historically relevant* special cases are called "equal Rabi" and "harmonic" because they correspond to the simplifications $\Omega_{m}=\Omega_{1}$, and $\Omega_{m}=\sqrt{m} \Omega_{1}$. The first obviously means all Rabi frequencies have been made equal. This is possible in atomic excitation. Transition wavelengths are quite different from one transition to the next in an atomic excitation chain, so different lasers are needed to obtain the required separate resonances, and the laser intensities can be adjusted in such a way that the field strengths compensate for differences in dipole matrix elements among the transitions, leading to equal $\Omega$ 's for every transition and thus equal elements in the Jacobi matrix. The second special case corresponds to excitation of a vibrational ladder of molecular states. In this case, the transition wavelengths are equal (if the vibration is purely harmonic) and only a single laser is needed to effect all transitions, so the $\sqrt{m}$ progression in the molecule's dipole matrix elements $\langle m+1|x| m\rangle$ will be present in the Jacobi matrix and reflected in its determinantal solutions.

Even in these two simplified special cases the eigenfrequencies obtained from the Jacobi determinant are generally incommensurate, but despite this there were found [2] consistent patterns of behavior in the atomic level probabilities as a function of time, and the patterns attain a close similarity for larger $N$. This is indicated in Fig. 2 for the equal-Rabi case. One conclusion is that, independent of the specific value of $N$, it is reasonable to expect the excitation of probability out of the ground level to proceed sequentially through the lower levels to the highest with a time lag from level to level.

*In the 1970's attention was called to problems associated with multi-level excitation of both atoms and molecules due to attempts to improve isotopic selectivity in laser-induced ionization and dissociation processes. 


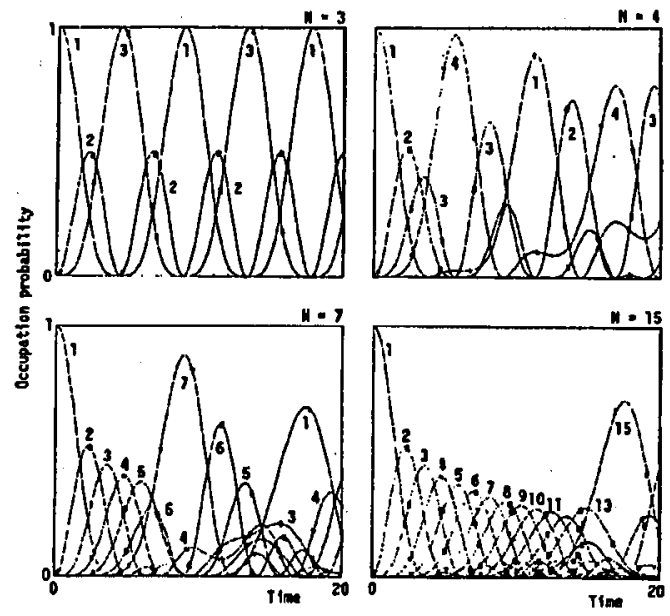

Fig. 2. Probability of excitation of the levels in several $N$-level systems, with the initial state labelled 1 (from Ref. [2a]). Note the general tendency for probability to flow from the initial level to the opposite end of the "chain" and then return, with the highest probability reached only at the ends of the chain.

With reference to the title of this paper, we have now reviewed the "short" aspects of $N$-level light-matter interactions. That is to say, we have not introduced any physical distance into our discussion at all. To treat the "long" aspects we must consider the propagation of the laser light. Thus we will next treat multi-level excitation during propagation of multiple laser pulses through a volume in which $N$-level atoms are dispersed in the manner of a uniform absorbing dielectric medium. The first modern attempts to combine coherent atomic response with on-resonance pulse propagation were successfully completed by $\mathrm{McCall}$ and IIahn [3], who at the same time discovered a deeper significance for pulse "area" as defined in (1).

Propagation requires that the dipole moments of the atoms be coupled with the light fields fully dynamically. That is, up to now the atoms were only allowed to respond to external fields, and the field amplitudes were fixed and could not respond to the atoms. A fully self-consistent interaction that accounts for the reaction of the fields to the atoms requires the introduction of Maxwell's wave equation in addition to Schrödinger's equation (3). Suppose that we begin with a general $N$-level atom. Then we may need $N-1$ laser fields, as mentioned already, and the Maxwell equations for them can be written in terms of their respective Rabi frequencies (since $\Omega$ is proportional to $\mathcal{E}$ ). These Maxwell equations will be only first-order equations [1] for the $\Omega$ 's, since the resonance condition eliminates the need to be concerned with the rapidly varying carrier wave (and thus the second derivatives in the wave equation), just as the RWA eliminated the rapid atomic transition frequencies from appearing in (3). Thus we can write the Maxwell 
contribution to the theory in the form

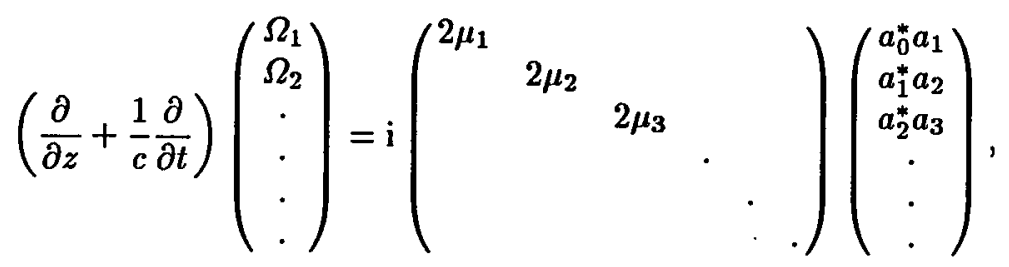

where the $\mu$ 's are the propagation constants proportional to the density of atoms and the transition oscillator strengths, as usual [1].

It is well known, following the pioneering work of McCall and Hahn [3], that the two-level self-consistent solution of these equations for a single propagating field is provided by a pulse of the form

$$
\Omega_{1}(z, t)=2 K V \operatorname{sech} K(z-V t),
$$

where $V$ is obviously the pulse velocity and $K V$ is the inverse pulse length. The propagation constant $\mu_{1}$ connects the velocity and the pulse length: $1 / V-1 / c=$ $\mu_{1} / 2(K V)^{2}$. We note that this pulse solution shows no attenuation. It has the form of a solitary wave, and is in fact a soliton. This is traditionally called a " $2 \pi$ pulse" because the total area, according to the infinite-time limit of (1), is just $2 \pi$. It is easy to understand the physical significance of the $2 \pi$ condition, because a $2 \pi$ rotation of the atom's Bloch vector rotates the atomic state back to its initial state at the end of the pulse. Speaking anthropomorphically about the pulse, it requests the atoms, as it passes through the medium, to radiate light in just such a way as to replace in its trailing edge whatever was absorbed from its leading edge in order to maintain its shape. In return for this favor, it takes nothing in the end from the atoms.

Now the question is, what happens in the $N$-level case? It is clear that the coupling of the Schrödinger equation (3) with the Maxwell equation (5) leaves valid the Jacobi form that was considered in the "short" form of our problem. However, the Maxwell equations act as a kind of constraint on the Jacobi form, and a nonlinear constraint at that.

An approach that can be taken is somewhat analogous to what was followed before. That is, we can look for eigenvalues in a generalized sense. Since the equations are nonlinear we do not expect to successfully impose a uniform time dependence like $\exp (-\mathrm{i} \lambda t)$, which was done to obtain the Jacobi recurrence formula. However, after some inspection, coupled with some experience, it is possible to see that there are space-time dependences that will do the job. That is, they will reduce (3) and (5) together to a set of coupled algebraic equations. Of course these coupled equations are nonlinear, and not amenable to the Jacobi analysis. Again following Einstein's advice we first simplify things somewhat by taking the various propagation constants $\mu$ to be equal. This need not be a severe approximation in many cases, and indeed cases involving only a few levels exist where it is not an approximation at all. One procedure we have identified asserts that all variables behave "the same" in the sense that their space-time dependence is through the single independent variable $Z \equiv z-V t$, as in the McCall-Hahn pulse (6). Then the simplest working rules for a solution are: 

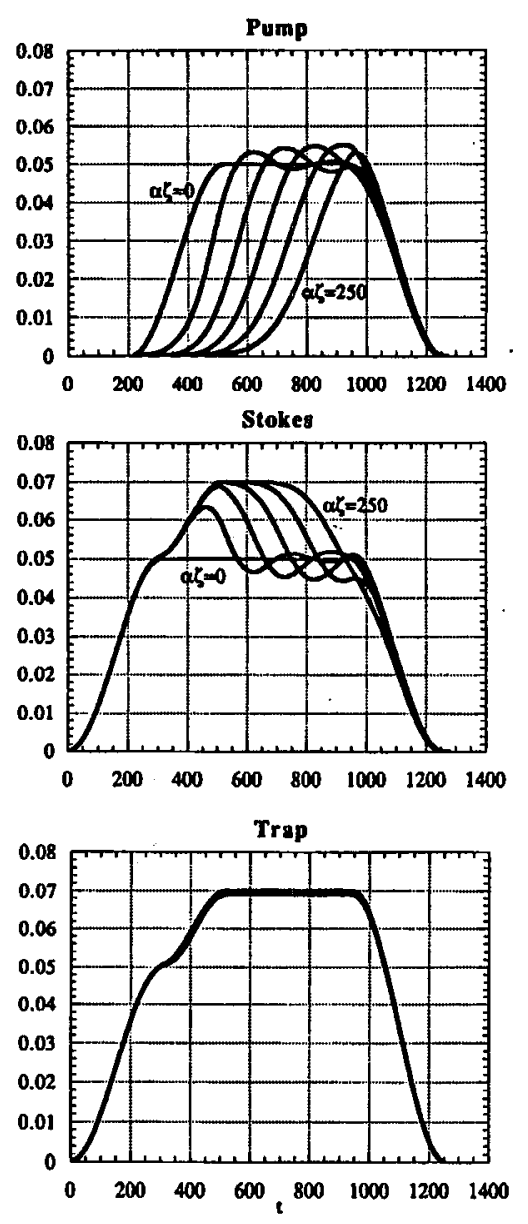

Fig. 3. Numerical solutions of the coupled Schrödinger and Maxwell equations (3) and (5) for the propagation of on-resonant pump and Stokes pulses in a Raman-type medium (see middle sketch in Fig. 1 for the type of atom). For greater realism a slight atomic relaxation has been included in the numerical evaluations. The intermediate level has been assumed to decay (out of the 3-level system) at a rate equal to 0.08 times the value of the peak Rabi frequencies, here taken equal for the pump and Stokes pulses (units can be taken as $\mathrm{GHz}$ ). The pump pulse is injected with a small delay (time in units of ps) after the Stokes pulse, to simulate the leading-edge delay between $\Omega_{1}$ and $\Omega_{2}$ in the idealized pulse solutions given in (7). The graphs show the changes in the pulses as they propagate. The top graph shows some absorption of the pump pulse, and the middle graph shows the corresponding amplification of the Stokes pulse. The bottom graph shows the "dressed field" combination (9) of the same pulses, and its strong resistance to any change at all. This confirms that the remark below (9) applies in contexts more general than the idealized solutions (7)-(8). 
(a) the field responsible for the first transition (called $\Omega_{1}$ above) is proportional to $\operatorname{sech} K Z$,

(b) all other fields are proportional to $\tanh K Z$,

(c) the atomic probability amplitude for the first level is equal to $\tanh K Z$, and

(d) all other probability amplitudes are proportional to $\operatorname{sech} K Z$.

There is not space to pursue a general situation, or to examine several deviations from this set of rules that appear to be possible, so we will give here the explicit solution to the simplest new case, a three-level atom (recall the middle sketch in Fig. 1) being excited by two fields. The elementary solutions are

$$
\begin{aligned}
& a_{0}=\tanh K Z, \quad a_{1}=\mathrm{i} d_{1} \operatorname{sech} K Z, \quad a_{2}=d_{2} \operatorname{sech} K Z, \\
& \Omega_{1}=M_{1} \operatorname{sech} K Z, \quad \Omega_{2}=M_{2} \tanh K Z,
\end{aligned}
$$

and after solving the nonlinear equations for the algebraic amplitudes we obtain

$$
\begin{aligned}
& d_{1}=-2 K V / M_{1}, \quad d_{2}=-M_{2} / M_{1}, \\
& \left(M_{1}\right)^{2}=\left(M_{2}\right)^{2}+(2 K V)^{2}, \quad(1 / V-1 / c)=2 \mu /\left(M_{1}\right)^{2} .
\end{aligned}
$$

It is a useful exercise to check two things: that the probabilities associated with all three levels add to 1 for all $z$ and $t$, and that when the third level amplitude and second field are removed, the previous one-field solution (6) of McCall and IIahn is recovered. In regard to pulse areas we note that the tanh pulse is what is called a $0-\pi$ pulse since its total area obviously vanishes. The sech pulse has its area determined by the "initial conditions," as in the McCall-IIahn case, but here in addition to the pulse length $(K V)^{-1}$ the initial conditions for $\Omega_{1}$ include the amplitude of $\Omega_{2}$, which is already well established long before $\Omega_{1}$ arrives on the scene.

This solution to an $N$-level propagation problem is an opening into questions associated with the "long" part of the title of this paper. It appears to provide new insights into the nature of multiple pulse propagation, and if the present pulse solutions are to be called simultons [4] (probably they should), they are clearly a new class of them. It is not clear to what extent the procedure for more general "long" solutions can be controlled in such a nice way as the Jacobi forms provided for the "short" solutions. However, one notes that a similarly "universal" behavior is predicted by rules (a)-(d) for the long response as was found for the short response [2], i.e., the response becomes qualitatively independent of the number of levels $N$ as soon as $N$ is large enough.

Finally, it is amusing to note that these solutions provide an ideal example of "dressed fields", which have recently been identified [5] in analogy to dressed states of atoms. In the present example, there are two dressied fields, according to the prescription that has been advanced [5]. The most interesting of these is denoted $\Omega_{-}$and it is defined as follows:

$$
\Omega_{-}(z, t)=a_{0} \Omega_{2}-a_{2} \Omega_{1} \text {. }
$$

A quick check, using (7) and (8), shows that $\Omega_{-}$is strictly constant. Figure 3 shows an example of a dressed field undergoing propagation in a three-level system. It 
is only very crudely similar to the solution sets given here, but it is nevertheless obeying what appears to be the principle of dressed field propagation in three-level systems - the dressed field (9) is almost completely invariant under space evolution.

\section{Acknowledgments}

This paper is submitted as a greeting and a token of my participation in the celebration of the 60th birthday of an old friend, Professor Iwo Bialynicki-Birula. The assistance of B.W. Shore, H.R. Haq and Liwei Wang in creating the figures for publication is gratefully acknowledged. Financial support in the research reported here was received from the US National Science Foundation through the Physics Division and the International Programs Division.

\section{References}

[1] L. Allen, J.H. Eberly, Optical Resonance and Two-Level Atoms, Dover, New York 1987, Chps. 2-4.

[2] (a) J.H. Eberly, B.W. Shore, Z. Białynicka-Birula, I. Białynicki-Birula, Phys. Rev. $A$ 16, 2038 (1977); and (b) Z. Bialynicka-Birula, I. Białynicki-Birula, J.H. Eberly, B.W. Shore, Phys. Rev. A 16, 2048 (1977).

[3] S.L. McCall, E.L. Hahn, Phys. Rev. 183, 457 (1967).

[4] M.J. Konopnicki, J.H. Eberly, Phys. Rev. A 24, 2567 (1981).

[5] J.H. Eberly, M.L. Pons, H.R. Haq, Phys. Rev. Lett. 72, 56 (1994). 